\title{
Robust Model Matching for Geometric Fault Detection Filters
}

\author{
Peter Seiler, József Bokor, Balint Vanek, and Gary J. Balas
}

\begin{abstract}
Geometric fault detection and isolation filters are known for having excellent fault isolation properties. However, they are generally assumed to be sensitive to model uncertainty and noise. This paper proposes a robust model matching method to incorporate model uncertainty into the design of geometric fault detection filters. Several existing methods for robust filter synthesis are described to solve the robust model matching problem. It is then shown that the robust model matching problem has an interesting self-optimality property for multiplicative input uncertainty models. Finally, a simple example is presented to study the effect of parametric uncertainty and unmodeled dynamics on the performance of a geometric filter.
\end{abstract}

\section{INTRODUCTION}

Fault tolerance is vital to ensuring the integrity and availability of safety critical systems. A fault tolerant system must also include the logic and algorithms for fault detection, fault diagnosis, fault containment, and reconfiguration to continue operation in face of failures. Filters to detect and isolate faults from system measurements form a key component of fault tolerant systems. The basic requirements for fault detection and isolation (FDI) filters typically include the following:

- Capability to isolate faults that occur simultaneously.

- Sensitivity to a particular fault and insensitivity to other faults.

- Robustness to modeling uncertainty.

- Good disturbance attenuation of external disturbances and noises

One popular FDI filter design technique, originally proposed by Massoumnia and Willsky [17], is based on geometric techniques. The original method was developed for systems with no disturbance or model uncertainty. As the name suggests, the geometric filter design technique exploits geometric properties of the system state-space. If the faults in the system have non-parallel signature directions then it is possible to design operators and output mixing maps that project the faults into disjoint, mutually orthogonal subspaces. The filter spectrum can be tuned to obtain desired transient response to faults. The geometric filter design paradigm was further extended in subsequent works to many different classes of systems [6], [15], [14], [9], [4], [2]. $\mathcal{H}_{2}$ and $\mathcal{H}_{\infty}$ model matching approaches to FDI filter design are also popular [23], [16], [10], [25], [13].

The basic requirements for FDI filters are often in conflict and a particular design approach can typically satisfy only a

P. Seiler and G.J. Balas are with the Aerospace and Engineering Mechanics Department, University of Minnesota, seiler@aem. umn. edu and balas@umn . edu.

B. Vanek and J. Bokor are with the Systems and Control Laboratory, Computer and Automation Research Institute, Hungarian Academy of Sciences, vanekesztaki.hu and bokoresztaki.hu. subset of the requirements. The geometric design approach, for example, is known for its excellent fault isolation, fault reconstruction and sensitivity properties under small modeling uncertainty and noise. However it is assumed to be sensitive as the model uncertainty and noise increase. This paper proposes a method that incorporate model uncertainty into the design. First, a geometric filter is designed on the nominal plant. Next a robust model matching problem is solved to design a filter that robustly matches the performance of the geometric filter over the set of uncertain plants. Several existing methods for robust filter synthesis are described to solve the robust model matching problem. It is then shown that the robust model matching problem has an interesting self-optimality property for multiplicative input uncertainty sets. Specifically, the filter designed on the nominal plant is the optimal filter in the robust model matching problem. Finally, a simple example is presented to study the effect of parametric uncertainty and unmodeled dynamics on the performance of a geometric filter.

\section{NOTATION}

$\mathbb{R}$ and $\mathbb{C}$ denote the set of real and complex numbers, respectively. $\mathbb{R} \mathbb{H}_{\infty}$ denotes the set of proper, rational functions with real coefficients that are analytic in the closed right half of the complex plane. $\mathbb{R}^{m \times n}, \mathbb{C}^{m \times n}$, and $\mathbb{R H}_{\infty}^{m \times n}$ denote the sets of $m \times n$ matrices whose elements are in $\mathbb{R}, \mathbb{C}$, and $\mathbb{R} \mathbb{H}_{\infty}$, respectively. A single superscript index is used to denote vectors, e.g. $\mathbb{R}^{l}$ denotes the set of $l \times 1$ vectors whose elements are in $\mathbb{R}$. For a matrix $M \in \mathbb{C}^{m \times n}, M^{*}$ denotes the complex conjugate transpose. $\bar{\sigma}(M)$ and $\underline{\sigma}(M)$ denote the maximum and minimum singular values. $\|M\|$ denotes the matrix norm induced by the vector 2-norm. It is known that $\|M\|=\bar{\sigma}(M)$. For a vector $v \in \mathbb{C}^{n}, \operatorname{Re}[v]$ denotes the real part of $v$. For $G \in \mathbb{R} \mathbb{H}_{\infty}^{m \times n},\|G\|_{\infty}:=\sup _{\omega} \bar{\sigma}(G(j \omega))$. Finally, let $G \in \mathbb{R H}_{\infty}^{(n+k) \times(n+m)}$ and $\Delta \in \mathbb{R} \mathbb{H}_{\infty}^{n \times n}$ be given and partition $G:=\left[\begin{array}{ll}G_{11} & G_{12} \\ G_{21} & G_{22}\end{array}\right]$ with $G_{11} \in \mathbb{R} \mathbb{H}_{\infty}^{n \times n}$ and $G_{22} \in \mathbb{R H}_{\infty}^{k \times m}$. If $I-G_{11} \Delta$ is invertible at $\omega=\infty$, then define $F_{u}(G, \Delta)$ as the linear fractional transformation (LFT) obtained by closing $\Delta$ around the upper channels of $G$ :

$$
\begin{gathered}
F_{u}(G, \Delta):=G_{22}+G_{21} \Delta\left(I-G_{11} \Delta\right)^{-1} G_{12} \\
\text { III. GEOMETRIC FDI FILTERS }
\end{gathered}
$$

This section briefly describes the formulation of fault detection filters designed using geometric concepts. The derivation of the geometric FDI filters is presented for LTI systems with no disturbance, no uncertainty and the detection and isolation of two faults. Consider the LTI system with two 
additive actuator faults:

$$
\begin{aligned}
& \dot{x}(t)=A x(t)+B u(t)+L_{1} f_{1}(t)+L_{2} f_{2}(t) \\
& y(t)=C x(t)
\end{aligned}
$$

where $L_{1}$ and $L_{2}$ represent the faults directions in the state space. $f_{1}$ and $f_{2}$ are the fault signals. The fault signals are zero if there is no fault but nonzero if the particular fault occurs. Only actuator faults are considered here but sensor faults can also be considered within the theory. The fundamental problem of residual generation is to synthesize residual generators (filters) with outputs $r_{i}(i=1,2)$ that have the following decoupling property: $r_{i}$ is sensitive to $f_{i}$ but insensitive to $f_{j}, i \neq j$. More precisely, if $f_{i}=0$ then $\lim _{t \rightarrow \infty} r_{i}(t)=0$ and if $f_{i} \neq 0$ then $r_{i} \neq 0$.

The solution of this problem depends on the $(C, A)$ invariant subspaces and certain unobservability subspaces [17]. A $(C, A)$-unobservability subspace $\mathcal{S}$ is a subspace such that there exist matrices $G$ and $H$ with the property that $\mathcal{S}$ is the maximal $(A+G C)$ invariant subspace contained in Ker $H C$. The family of $(C, A)$-unobservability subspaces containing a given set $\mathcal{L}$ has a minimal element. Define $\mathcal{L}_{i}=\mathrm{Im}$ $L_{i}(i=1,2)$ and denote by $\mathcal{S}^{*}$ the smallest unobservability subspace containing $\mathcal{L}_{2}$. Then the fundamental problem of residual generation has a solution if and only if $\mathcal{S}^{*} \cap \mathcal{L}_{1}=0$ [17]. The condition $\mathcal{S}^{*} \cap \mathcal{L}_{1}=0$ ensures that the fault to be detected is not hidden in the unobservability subspace of the detection filter. In fact, the fault direction will be decoupled from the rest of the fault directions since they are contained in the unobservability subspace of the residual generator. This result can be extended to LPV systems [5].

The residual generator associated with fault direction $L_{1}$ can be described by an observer of the form:

$$
\begin{aligned}
\dot{w}(t) & =N w(t)-G y(t)+F u(t) \\
r_{1}(t) & =M w(t)-H y(t)
\end{aligned}
$$

where $u$ and $y$ are the known input and measured output signals of the original LTI system. $w$ is the state of the residual generator and $r_{1}$ is the residual.

Denote by $P$ the projection operator $P: \mathcal{X} \rightarrow \mathcal{X} / \mathcal{S}^{*}$. The state matrices can be determined as follows [17]. $H$ is a solution of the equation $\operatorname{Ker} H C=\operatorname{Ker} C+\mathcal{S}^{*}$, and $M$ is the unique solution of $M P=H C$. Consider a gain matrix $\hat{G}$ chosen such that $(A+\hat{G} C) \mathcal{S}^{*} \subseteq \mathcal{S}^{*}$ and define $\hat{A}=P(A+G C) P^{T} . \hat{A}$ is not necessarily Hurwitz. To obtain quadratically stable filters one can set $N=\hat{A}+\tilde{G} M$, where $\tilde{G}:=X^{-1} K$ and $X, K$ are determined from the linear matrix inequality (LMI):

$$
\begin{aligned}
& 0 \succeq \hat{A}^{T} X+X \hat{A}+M^{T} K^{T}+K M \\
& 0 \preceq X=X^{T}
\end{aligned}
$$

Then set $G=P \hat{G}+\tilde{G} H$ and $F=P B$.

Using this approach there are as many filters as faults to detect, and their state dimensions are equal to the dimension of $\mathcal{X} / \mathcal{S}^{*}$. The filter poles can be tuned by imposing constraints in the LMI resulting in perfect reconstruction of fault signals $f_{i}$. One issue is that the filter design does not consider model uncertainty and the fault detection performance may not be robust. The next section discusses a model matching approach for recovering the geometric filter performance in the presence of model uncertainty.

\section{Robust Model Matching}

This section considers a robust model matching problem for geometric filter design on uncertain plants. Then several existing methods for robust filter synthesis are described. The final subsection shows that the robust model matching problem has an interesting self-optimality property for multiplicative input uncertainty sets.

\section{A. Problem Formulation}

Let $G_{u}$ denote an uncertain plant for which the filter will be designed. The standard linear fractional transformation (LFT) framework [19] can be used to model the uncertainties. Let $G \in \mathbb{R} \mathbb{H}_{\infty}^{(n+k) \times(n+m)}$ and $\boldsymbol{\Delta} \subseteq \mathbb{R} \mathbb{H}_{\infty}^{n \times n}$ be given. ${ }^{1}$ Define the set of models

$$
\mathcal{M}:=\left\{G_{u}=F_{u}(G, \Delta): \Delta \in \Delta,\|\Delta\|_{\infty} \leq 1\right\}
$$

It is assumed that $F_{u}(G, \Delta)$ is well defined for all $\Delta \in \Delta$ with $\|\Delta\|_{\infty} \leq 1 . \Delta$ is typically a set describing a block structured system that can include (repeated) real parametric and LTI dynamic system uncertainties. Nonlinear and/or time-varying uncertainties can also be modeled using integral quadratic constraints [18]. The restriction that $\Delta$ be a square system is only for notational simplicity.

Each $G_{u} \in \mathcal{M}$ is a system that relates the faults and plant inputs to the signals available to the fault detection filter:

$$
\left[\begin{array}{l}
y \\
u
\end{array}\right]=G_{u}\left[\begin{array}{l}
f \\
u
\end{array}\right]
$$

The objective is to design a filter $F$ with inputs $\left[\begin{array}{l}y \\ u\end{array}\right]$ and output residuals $r$ such that the residuals have "good" fault decoupling properties for all models $G_{u} \in \mathcal{M}$.

A robust model matching problem is now described to meet this objective. The nominal plant in the set $\mathcal{M}$ is given by $\Delta=0$, i.e. $G_{0}:=F_{u}(G, 0)$ is the nominal plant. First, design a geometric filter $F_{0}$ to solve the fundamental problem of residual generation on the nominal plant $G_{0}$. The model matching method attempts to design a filter $F$ such that the performance on the uncertain plant $G_{u}$ robustly matches the designed behavior of $F_{0} G_{0}$. Mathematically, the proposed design problem is:

Problem 1: Let $G \in \mathbb{R} \mathbb{H}_{\infty}^{(n+k) \times(n+m)}, \boldsymbol{\Delta} \subseteq \mathbb{R H}_{\infty}^{n \times n}$ and $F_{0} \in \mathbb{R H}_{\infty}^{l \times k}$ be given. The robust model matching problem is:

$$
\min _{F \in \mathbb{R}_{\infty}^{l \times k}} \max _{G_{u} \in \mathcal{M}}\left\|F_{0} G_{0}-F G_{u}\right\|_{\infty}
$$

The interconnection for this robust model matching problem is shown in shown in Figure 1. The reference model is given by $F_{0} G_{0}$. The nominal residual response $r_{0}$ will have

\footnotetext{
${ }^{1} G$ and $F$ were used in the previous section to denote gain matrices in the geometric filter. In this section $G$ and $F$ will denote systems in the model matching design.
} 
the desired decoupling properties given by the fundamental problem of residual generation. The optimization in Equation 8 designs a filter $F$ that most closely matches, in a worstcase sense, the desired residual generation behavior $F_{0} G_{0}$. In this paper the focus is on fault detection filters designed using the geometric approach but the model matching problem can, in principle, be used to robustly match the behavior of any filter $F_{0}$ designed on the nominal system $G_{0}$. It is worth to note, that other methods dealing with the uncertain system directly like [8], obtain different FDI solution for a different problem, which might lead to better overall performance.

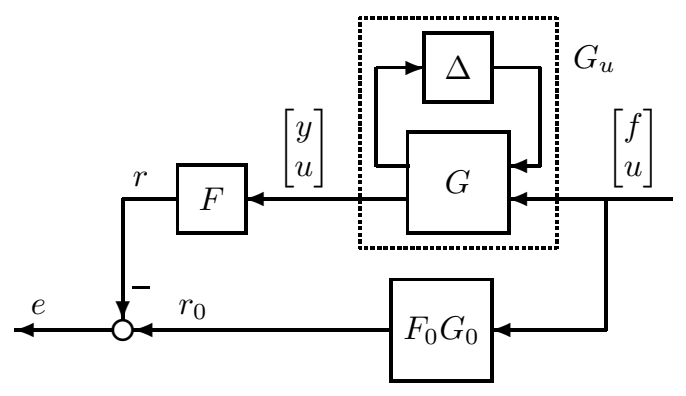

Fig. 1. Robust model matching

\section{B. Filter Synthesis}

There are several approaches to solve the robust model matching problem. Sun and Packard observed that robust filter design (Equation 8) is an infinite-dimensional convex optimization in the filter [22]. They developed an algorithm to compute the globally optimal robust filter for the special case where $\boldsymbol{\Delta}$ only models repeated real uncertainties [22]. It does not seem possible to extend this algorithm to sets $\Delta$ that include dynamic uncertainties, nonlinearities and/or time-varying operators.

The standard approach to handle more complicated uncertainty sets is to replace $\max _{G_{u} \in \mathcal{M}}\left\|F_{0} G_{0}-F G_{u}\right\|_{\infty}$ with an upper-bound. For example, when $\Delta$ contains only LTI uncertainty the maximization over $\mathcal{M}$ can be replaced with the $\mu$ upper bound which involves a minimization over $D$ scales [19]. The design problem can then be recast as a $\mu$ synthesis problem involving a search for the filter and the $D$ scales. $\mu$-synthesis is, in general, a nonconvex problem and the coordinate-wise $\mathrm{D}-\mathrm{K}$ iteration has been applied to solve for the filter and uncertainty multipliers [1]. The D$\mathrm{K}$ iteration yields sub-optimal solutions but is a standard method to handle the nonconvexity that arises in robust control synthesis.

In robust filter design problem, the filter enters the design interconnection in an open loop (rather than a feedback) configuration and this structure can be exploited. There are two different approaches to convert the $\mu$-synthesis problem into an infinite dimensional convex optimization problem ([20] and [21]). Both approaches use the more general IQC framework to model the uncertainty and obtain an upper bound on the worst-case performance. In [20], the filter synthesis problem is converted into an infinitedimensional (convex) semi-definite program (SDP) [7]. The set of allowable IQC multipliers is infinite dimensional and a finite dimensional optimization is obtained by restricting the multipliers to be a combination of chosen basis functions. In [21], the robust filter design problem is turned into a frequency-dependent, infinite dimensional linear matrix inequality (LMI) in the filter and multipliers. Next, a finite dimensional optimization is obtained by enforcing the frequency-dependent LMI on a dense frequency grid and restricting the filter to be a linear combination of chosen basis functions. The frequency-dependent IQC multipliers are allowed to be arbitrary functions on the frequency grid. To summarize, the two approaches use roughly dual methods to convert the robust filter design problem to a finite dimensional convex optimization: In [21], basis functions are used for the filter but the multipliers (scalings) are allowed to be arbitrary functions on the frequency grid. In [20] basis functions are chosen for the multipliers but the filter is allowed to be an arbitrary, linear system.

The various methods to solve the robust filter design problem have benefits and drawbacks in terms of computational complexity and ease of formulating the problem (e.g. picking basis functions for the filter or for the uncertainty scalings). The next section shows that the robust model matching problem has an interesting self-optimality property for multiplicative input uncertainty sets. Specifically, $F_{0}$ itself is the optimal filter for this uncertainty structure.

\section{Multiplicative Input Uncertainty}

This section considers the robust model matching problem for input multiplicative uncertainty. The uncertain system is given by $G_{u}:=G_{0}(I+w \Delta)$ where $w \in \mathbb{R H}_{\infty}$ is a weight that specifies the level of uncertainty at each frequency by $|w(j \omega)| .|w(j \omega)|=1$ corresponds to $100 \%$ input uncertainty at frequency $\omega$ and hence weights typically satisfy $\|w\|_{\infty} \leq 1$. Input multiplicative uncertainty is a commonly used uncertainty model because the effect of uncertainty can be quickly assessed by choosing simple weights $w$, and it is sufficiently general since other uncertainty structures can be recast into input multiplicative form. For example, a reasonable uncertainty model is obtained by choosing $w$ to be a first order system with small magnitude at low frequencies and magnitude close to one at high frequencies, to represent that we have fairly good model of the system around steady state, while at high frequencies the system model is less accurate. Alternatively, the Matlab function ucover [3] can be used to compute a $w$ so that the uncertainty set $\mathcal{M}$ contains a given, finite set of LTI systems. The weight can generally be chosen as a full matrix but the result in this section is restricted to weights of the form $w(s) I$.

The design interconnection for the robust model matching problem with input multiplicative uncertainty is shown in Figure 2. $G_{0}$ again denotes the nominal system and $F_{0}$ is a filter that has been designed to achieve some desired performance on the nominal plant. For this uncertainty structure the robust model matching problem can be equivalently stated as: 
Problem 2: Let $F_{0} \in \mathbb{R H}_{\infty}^{m \times n}, G \in \mathbb{R H}_{\infty}^{n \times k}$ and $w \in$ $\mathbb{R H}_{\infty}$ be given. The robust model matching problem is:

$$
\min _{F \in \mathbb{R} \mathbb{H}_{\infty}^{m \times n}} \max _{\Delta \in \mathbb{R} \mathbb{H}_{\infty}^{k \times k},\|\Delta\|_{\infty} \leq 1}\left\|F_{0} G-F G(I+w \Delta)\right\|_{\infty}
$$

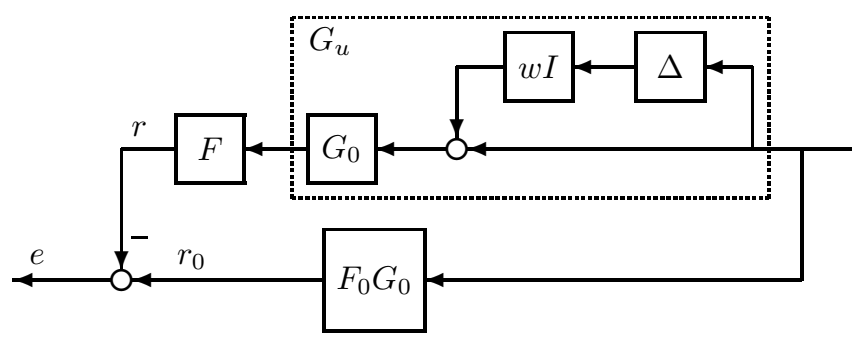

Fig. 2. Robust model matching with multiplicative input uncertainty

The next theorem presents the main result of this section.

Theorem 1: If $\|w\|_{\infty} \leq 1$ then $F_{0}$ is the optimal filter for the robust model matching problem.

Proof: The robust model matching problem can be equivalently written as:

$$
\min _{F \in \mathbb{R} \mathbb{H}_{\infty}^{m \times n}} \max _{\omega} \max _{\substack{\Delta \in \mathbb{R H}^{k \times} \\|\Delta(j \omega)| \leq|w(j \omega)|}}\left\|\left(F_{0} G-F G(I+\Delta)\right)(j \omega)\right\|
$$

The min-max is always greater than the max-min and hence a lower bound on the model matching problem is obtained by:

$$
\max _{\omega} \min _{F \in \mathbb{R} \mathbb{H}_{\infty}^{m \times n}} \max _{\substack{\Delta \in \mathbb{R}^{m} \boldsymbol{H}_{\infty} \times k \\|\Delta(j \omega)| \leq|w(j \omega)|}}\left\|\left(F_{0} G-F G(I+\Delta)\right)(j \omega)\right\|
$$

Next, the constraints that $F$ and $\Delta$ be stable are dropped:

$\max _{\omega}\left[\min _{F \in \mathbb{C}^{m \times n}} \max _{\substack{\Delta \in \mathbb{C}^{k^{*}} \\|\Delta| \leq|w(j \omega)|}}\left\|\left(F_{0} G\right)(j \omega)-F G(j \omega)(I+\Delta)\right\|\right]$

The max over $\Delta$ is unchanged by dropping the stability constraint but the min over $F$ is potentially lower once we drop the stability constraint. Thus the result of Equation 11 is no greater than the optimal value for Equation 10 .

Next, apply Lemma 2 in the appendix with $A:=$ $F_{0}(j \omega), B:=G(j \omega)$, and $\alpha:=|w(j \omega)|$. By this lemma and the assumption $\|w\|_{\infty} \leq 1$, the optimization in the brackets of Equation 11 has an optimal cost equal to $|w(j \omega)|\left\|\left(F_{0} G\right)(j \omega)\right\|$ at each $\omega$ and the optimal value is achieved by $F=F_{0}(j \omega)$.

Thus the optimal cost for the robust model matching problem is lower bounded by $\left\|w F_{0} G\right\|_{\infty}$. This cost is achieved by the choice $F=F_{0}$ and hence $F_{0}$ is the optimal filter.

Roughly, this result implies that the robust model matching filter design is self optimal for this input multiplicative uncertainty set. The uncertainty degrades the performance but it does so in a way that apparently cannot be exploited by any other filter. Note that this result is not specific to nominal filters $F_{0}$ designed with the geometric method. The result only depends on the formulation of the robust model matching problem and the specific structure of the input multiplicative uncertainty.

\section{EXAMPLE}

Consider the fault detection example from [11], [24]. The nominal fault system $G_{0}$ has the form of Equation 2 with state space matrices given by:

$$
\begin{aligned}
A & =\left[\begin{array}{ccc}
-0.8 & 0 & 0 \\
0 & -0.5 & 0.6 \\
0 & -0.6 & -0.5
\end{array}\right], B=\left[\begin{array}{ll}
1 & 1 \\
1 & 0 \\
0 & 1
\end{array}\right] \\
L_{1} & =\left[\begin{array}{l}
1 \\
1 \\
0
\end{array}\right], L_{2}=\left[\begin{array}{l}
1 \\
0 \\
1
\end{array}\right], C=\left[\begin{array}{lll}
0 & 1 & 1 \\
1 & 1 & 0
\end{array}\right]
\end{aligned}
$$

The following FDI filter $F_{0}$ was designed to generate fault residuals for this system using the geometric method:

$$
\begin{aligned}
\dot{w}(t) & =\left[\begin{array}{cccc}
-1.10 & 0.48 & 0 & 0 \\
-0.64 & -3.90 & 0 & 0 \\
0 & 0 & 0.390 & 1.89 \\
0 & 0 & -5.89 & -8.22
\end{array}\right] w(t) \\
& +\left[\begin{array}{cc}
0.26 & 0.34 \\
2.40 & -2.19 \\
-1.47 & 0.84 \\
7.73 & -4.16
\end{array}\right] y(t)+\left[\begin{array}{cc}
1.00 & 0 \\
-0.71 & 0 \\
0 & -0.71 \\
0 & 1.00
\end{array}\right] u(t) \\
{\left[\begin{array}{l}
r_{1}(t) \\
r_{2}(t)
\end{array}\right] } & =\left[\begin{array}{cccc}
0 & 3.25 & 0 & 0 \\
0 & 0 & -5.66 & -8.01
\end{array}\right] w(t)+\left[\begin{array}{cc}
-2.30 & 2.30 \\
8.01 & -4.00
\end{array}\right] y(t)
\end{aligned}
$$

The Bode magnitude plots of $F_{0} G_{0}$ from faults $f$ to residuals $r$ is shown in Figure 3. The geometric filter demonstrates the desired decoupling properties. The transfer functions from $f_{i}$ to $r_{i}(i=1,2)$ have low pass characteristics with steady state gain of 1 . The response of $r_{i}$ to $f_{j}(j \neq i)$ is extremely small.
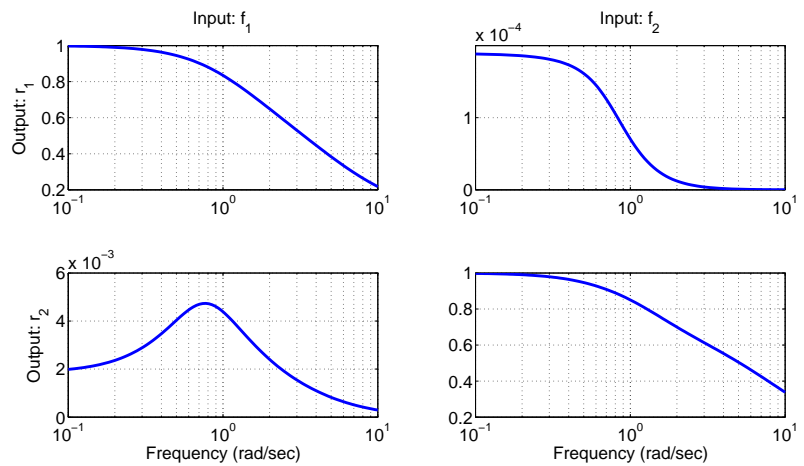

Fig. 3. Bode magnitude plot of nominal response from $f$ to $r$

The robust model matching approach described in Section IV applies to uncertainty structures that can be formulated within the LFT framework. The remainder of the section considers the effect of parametric uncertainty and unmodeled dynamics.

First, consider the effect of parametric uncertainty in the plant state matrix:

$$
A=\left[\begin{array}{ccc}
-0.8 & 0 & 0 \\
0 & -0.5\left(1+\delta_{1}\right) & 0.6\left(1+\delta_{2}\right) \\
0 & -0.6\left(1+\delta_{2}\right) & -0.5\left(1+\delta_{1}\right)
\end{array}\right],
$$


where $\left|\delta_{1}\right| \leq 0.1$ and $\left|\delta_{2}\right| \leq 0.1$. Let $\mathcal{M}$ denote the set of models described by this uncertainty. $\mathcal{M}$ can be described by an LFT $G_{u}=F_{u}(G, \Delta)$ as in Equation 6. The performance of the filter $F_{0}$ on any $G_{u} \in \mathcal{M}$ will deviate, in general, from the nominal. The dashed curve in Figure 4 shows the worst-case performance of $F_{0}$ :

$$
\max _{G_{u} \in \mathcal{M}}\left\|F_{0} G_{0}-F_{0} G_{u}\right\|_{\infty}
$$

Perfect matching of $F_{0} G_{0}$ would correspond to a gain of zero. The gain of the reference system $F_{0} G_{0}$ is approximately 1.0 at low frequencies (Figure 3 ). Thus the performance of $F_{0}$ degrades by approximately $33 \%$ over the uncertainty set. One of the filter synthesis methods described in Section IV-B could be applied to improve the robust matching of the nominal performance $F_{0} G_{0}$. However, it is possible to compute a lower bound on the optimal worst-case performance achieved by any filter [12], [21]. The solid curve in Figure 4 shows the lower bound on the best achievable filter performance. The nominal filter $F_{0}$ is very close to achieving the optimal performance and hence a robust filter synthesis will not yield significant improvements on the worst-case performance.

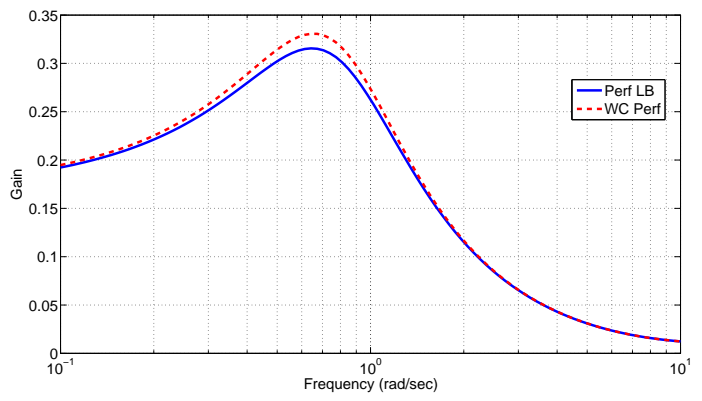

Fig. 4. Worst-case performance of $F_{0}$

Next consider the effect of input multiplicative uncertainty with the weight

$$
w(s):=\frac{s+0.1}{s+2}
$$

The parametric uncertainty in $A$ is not considered in this part of the analysis. This weight corresponds to $100 \%$ uncertainty at high frequencies and 5\% uncertainty at low frequencies. The dashed curve in Figure 5 shows the worstcase performance of $F_{0}$. The performance of $F_{0}$ degrades by approximately $51 \%$ over the uncertainty set. The solid curve in Figure 4 shows the lower bound on the best achievable filter performance. The two curves are equal as expected based on Theorem 1. Thus $F_{0}$ is the optimal filter for robustly matching its own performance on the nominal plant. In future work, it is our intention to compare the results obtained here with methods dealing with uncertain system description directly [8], to assess the drawback of formulating the robust FDI problem as a model matching problem.

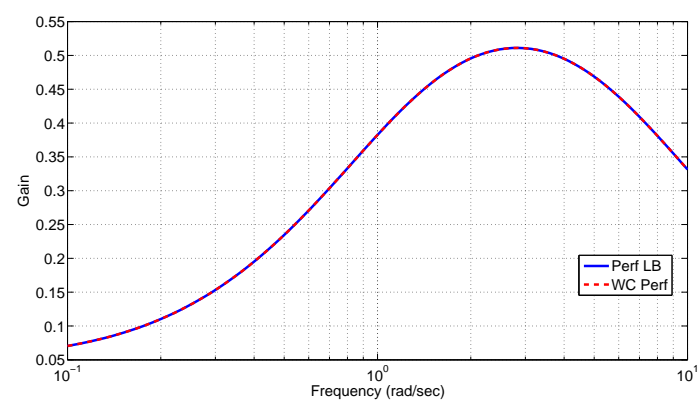

Fig. 5. Bode plot of optimal filter

\section{CONCLUSIONS}

This paper proposed a method incorporate model uncertainty into the design of geometric fault detection and isolation filters. First, a geometric filter is designed on the nominal plant. Next a robust model matching problem is solved to design a filter that robustly matches the performance of the geometric filter over the set of uncertain plants. Several existing methods for robust filter synthesis were described to solve the robust model matching problem. It was then shown that the robust model matching problem has an interesting self-optimality property for multiplicative input uncertainty sets. Finally, a simple example was presented to study the effect of parametric uncertainty and unmodeled dynamics on the performance of a geometric filter.

\section{ACKNOWLEDGMENTS}

This material is based upon work supported by the National Science Foundation under Grant No. 0931931 entitled "CPS: Embedded Fault Detection for Low-Cost, SafetyCritical Systems". Any opinions, findings, and conclusions or recommendations expressed in this material are those of the author(s) and do not necessarily reflect the views of the National Science Foundation.

\section{REFERENCES}

[1] B. Appleby, J. Dowdle, and W. VanderVelde. Robust estimator design using $\mu$ synthesis. In Proc. of the IEEE Conference on Decision and Control, pages 640-645, 1991.

[2] S. Armeni, A. Casavola, and E. Mosca. Robust fault detection and isolation for LPV systems under a sensitivity constraint. International Journal of Adaptive Control and Signal Processing, 23(1):55-72, 2008.

[3] G. Balas, R. Chiang, A. Packard, and M. Safonov. Robust Control Toolbox. MathWorks, 2010.

[4] G. J. Balas and J. Bokor. Detection filter design for LPV systems. $4^{\text {th }}$ IFAC SAFEPROCESS Symposium, 2:653-656, 2000.

[5] J. Bokor and G. Balas. Detection filter design for LPV systems - A Geometric approach. Automatica, 40:511-518, 2004.

[6] J. Bokor, A. Edelmayer, and L. Keviczky. An $\mathcal{H}_{\infty}$ filtering approach to robust detection of failures in dynamical systems. Proc. 33rd IEEE Conf. on Decision and Control, 3:3037-3039, 1994.

[7] S. Boyd, L. El Ghaoui, E. Feron, and V. Balakrishnan. Linear Matrix Inequalities in System and Control Theory, volume 15 of Studies in Applied Mathematics. SIAM, 1994.

[8] F. Castang, A. Zolghadri, D. Henry, and M. Monsion. A lmi approach for designing robust fdi filters with guaranteed fault sensitivity performance. In Systems, Man and Cybernetics, 2002 IEEE International Conference on, page 6 pp. vol.7, 2002. 
[9] C. De Persis and A. Isidori. A geometric approach to nonlinear fault detection and isolation. IEEE Transactions on Automatic Control, 46(6):853-865, 2001.

[10] Steven X. Ding. Model-based Fault Diagnosis Techniques. Springer, 2008.

[11] A. Edelmayer and J. Bokor. Optimal $H_{\infty}$ scaling for sensitivity optimization of detection filters. International Journal of Robust and Nonlinear Control, 12:749-760, 2002.

[12] A. Giusto and F. Paganini. Robust synthesis of feedforward compensators. IEEE Trans. on Automatic Control, 44(8):1578-1582, 1999.

[13] Emmanuel Mazarsand Imad M. Jaimoukha and Zhenhai Li. Computation of a reference model for robust fault detection and isolation residual generation. Journal of Control Science and Engineering, 2008:1-12, 2008.

[14] Michel Kinnaert. Robust fault detection based on observers for bilinear systems. Automatica, 35(11):1829-1842, 1999.

[15] R. S. Mangoubi. Robust Estimation and Failure Detection - A Concise Treatment. Springer-Verlag, London, 1998.

[16] A. Marcos, S. Ganguli, and G.J. Balas. An application of $\mathcal{H}_{\infty}$ fault detection and isolation to a transport aircraft. Control Engineering Practice, 13(1):105-119, 2005.

[17] M. A. Massoumnia. A geometric approach to the synthesis of failure detection filters. IEEE Trans. Automatic Control, AC-31(9):839-846, 1986.

[18] A. Megretski and A. Rantzer. System analysis via integral quadratic constraints. IEEE Trans. on Automatic Control, 42(6):819-830, 1997.

[19] A. Packard and J. Doyle. The complex structured singular value. Automatica, 29(1):71-109, 1993.

[20] C.W. Scherer and I.E. Köse. Robustness with dynamic IQCs: An exact state-space characterization of nominal stability with applications to robust estimation. Automatica, 44:1666-1675, 2008.

[21] P. Seiler, B. Vanek, J. Bokor, and G.J. Balas. Robust $H_{\infty}$ filter design using frequency gridding. In Proc. of the American Control Conference, 2011.

[22] K. Sun and A. Packard. Optimal, worst-case filter design via convex optimization. In Proc. of the IEEE Conference on Decision and Control, pages 1380-1385, 2003.

[23] A. Varga. On computing least order fault detectors using rational nullspace bases. In In Proceedings of the IFAC Symp. SAFEPROCESS'2003, Washington D.C., 2003.

[24] A. Varga. Integrated algorithm for solving $\mathrm{H}_{2}$-optimal fault detection and isolation problems. In Submitted to 2010 Conference on Control and Fault Tolerant Systems, 2010.

[25] A. Zolghadri, F. Castang, and D. Henry. Design of robust fault detection filters for multivariable feedback systems. International journal of modelling and simulation, ACTA Press, 26(1), 2006.

\section{APPENDIX}

Lemma 1: Let $c_{1}, c_{2} \in \mathbb{R}$ be non-negative constants. If $u, v \in \mathbb{C}^{n}$ and $\operatorname{Re}\left[u^{*} v\right] \geq 0$ then $\left\|c_{1} u\right\| \leq\left\|c_{1} u+c_{2} v\right\|$.

Lemma 2: Let $\alpha \in \mathbb{R}$ be a strictly positive constant and let $A \in \mathbb{C}^{m \times n}$ and $B \in \mathbb{C}^{n \times k}$ be given matrices. Define $J: \mathbb{C}^{m \times n} \rightarrow \mathbb{R}$ as:

$$
J(X):=\max _{\Delta \in \mathbb{C}^{k} x_{k}, \bar{\sigma}(\Delta) \leq \alpha}\|A B-X B-X B \Delta\|
$$

Then

$$
\min _{X \in \mathbb{C}} J(X)= \begin{cases}\alpha\|A B\| & \text { if } \alpha \leq 1 \\ \|A B\| & \text { if } \alpha>1\end{cases}
$$

The minimal cost is achieved by $X^{*}=A$ if $\alpha \leq 1$ and $X^{*}=0$ if $\alpha>1$.

Proof: Let $u \in \mathbb{C}^{k}$ and $v \in \mathbb{C}^{m}$ be the input/output vectors associated with the maximum singular value of $A B$, i.e. $u$ and $v$ satisfy $A B u=\bar{\sigma}(A B) v,\|u\|=1$, and $\|v\|=1$.

Assume $\alpha>1$ and pick any $X \in \mathbb{C}^{m \times n}$. If $R e\left[(X B u)^{*} v\right] \geq 0$ then choose $\Delta_{0}=-\alpha I . J(X)$ can be lower-bounded as:

$$
\begin{aligned}
J(X) & \geq\left\|A B-X B-X B \Delta_{0}\right\| \\
& =\|A B+(\alpha-1) X B\| \\
& \geq\|(A B+(\alpha-1) X B) u\| \\
& \geq\|\bar{\sigma}(A B) v+(\alpha-1) X B u\| \\
& \geq \bar{\sigma}(A B)
\end{aligned}
$$

The first inequality follows from the definition of $J(X)$ in Equation 19 while the equality follows from the definition of $\Delta_{0}$. The next two inequalities follow from the definition of the matrix norm (maximum singular value) and the choices of $u$ and $v$. The final inequality follows from Lemma 1 . If $\operatorname{Re}\left[(X B u)^{*} v\right] \leq 0$ then similar steps can be used to again show that $J(X) \geq \bar{\sigma}(A B)$ with the choice $\Delta_{0}=+\alpha I$. Thus $J(X) \geq \bar{\sigma}(A B)$ and the lower bound is achieved by $X=0$.

Next assume $\alpha \leq 1$. Pick any $X \in \mathbb{C}^{m \times n}$ and define $Y:=-A+X$. If $\operatorname{Re}\left[(Y B u)^{*} v\right] \geq 0$ then choose $\Delta_{0}=\alpha I$. Similar to the steps above, $J(X)$ can be lower-bounded as:

$$
\begin{aligned}
J(X) & \geq\|\alpha A B+(\alpha+1) Y B\| \\
& \geq\|\alpha \bar{\sigma}(A B) v+(\alpha+1) Y B u\| \\
& \geq \alpha \bar{\sigma}(A B)
\end{aligned}
$$

The first inequality follows from the choice of $\Delta_{0}$ and the definition of $Y$. The next inequality again follow from the choices of $u$ and $v$ while the final inequality follows from Lemma 1. If $\operatorname{Re}\left[(Y B u)^{*} v\right] \leq 0$ then similar steps can be used to show that $J(X) \geq \alpha \bar{\sigma}(A B)$ with the choice $\Delta_{0}=-\alpha I$. Thus $J(X) \geq \alpha \bar{\sigma}(A B)$ and the lower bound is achieved by $X=A$. 